Journal for ImmunoTherapy of Cancer

\section{Viral status, immune microenvironment and immunological response to checkpoint inhibitors in hepatocellular carcinoma}

To cite: Ho WJ, Danilova L, Lim SJ, et al. Viral status, immune microenvironment and immunological response to checkpoint inhibitors in hepatocellular carcinoma. Journal for ImmunoTherapy of Cancer 2020;8:e000394. doi:10.1136/ jitc-2019-000394

- Additional material is published online only. To view please visit the journal online (http://dx.doi.org/10.1136/jitc2019-000394).

Accepted 16 March 2020
Check for updates

(c) Author(s) (or their employer(s)) 2020. Re-use permitted under CC BY-NC. No commercial re-use. See rights and permissions. Published by BMJ.

For numbered affiliations see end of article.

Correspondence to

Mark Yarchoan;

mark.yarchoan@jhmi.edu

\section{ABSTRACT}

Background and aims Immune checkpoint inhibitors (ICls) targeting the programmed cell death protein 1 (PD-1)/programmed death-ligand 1 (PD-L1) pathway have clinical activity in hepatocellular carcinoma (HCC), but only a subset of patients respond to these therapies, highlighting a need for novel biomarkers to improve clinical benefit. HCC usually occurs in the setting of liver cirrhosis from chronic hepatitis B or C viral infection, but the effects of viral status on the tumor immune microenvironment and clinical responses to ICls in HCC remains unclear.

Methods We conducted a meta-analysis to estimate the objective response rates for PD-1/PD-L1 inhibitors in virally-infected and uninfected patients, and examined the effects of viral etiology on the tumor microenvironment using data from The Cancer Genome Atlas, as well as peripheral blood responses using an independent cohort of patients studied by mass cytometry (cytometry by time-offlight (CyTOF)).

Results Meta-analysis comparing objective response rates (ORR) between virally-infected and uninfected patients showed no clinically meaningful difference (absolute difference of ORR in virally-infected vs uninfected $=-1.4 \%, 95 \% \mathrm{Cl}$ : $-13.5 \%$ to $10.6 \%$ ). There was no relationship between viral etiology on features of the tumor immune microenvironment that are known to modulate responses to PD-1/PD-L1 inhibitors, and the tumor mutational burden was similar between virallyinfected and uninfected HCC. RNA sequencing of tissueresident $T$ cell and $B$ cell repertoires similarly showed no effect of viral status on their diversity. CyTOF analysis of peripheral blood specimens further demonstrated similar expression of immune-related markers in response to PD-1 inhibitor therapy in virally-infected and uninfected HCC.

Conclusion There is no significant effect of viral etiology on the tumor immune microenvironment in HCC, and viral status should not be used as a criterion to select patients for PD-1/PD-L1 therapy.

\section{BACKGROUND}

Immune checkpoint inhibitors (ICIs) targeting the programmed cell death protein 1 (PD-1)/programmed death-ligand 1
(PD-L1) pathway have broad clinical activity against a diverse array of tumors types. In hepatocellular carcinoma (HCC), inhibitors of the PD-1/PD-L1 pathway have consistently demonstrated objective response rates of $14 \%$ to $20 \%$ as monotherapy, and these responses are often durable. ${ }^{12}$ Multiple additional ICIs are now in clinical development, as monotherapy and in combination with other immunotherapies or targeted therapies. Despite clearly having activity in $\mathrm{HCC}$, recent phase 3 studies of PD-1 inhibitors have failed to meet their primary endpoints, highlighting a need for novel biomarkers to identify the subsets of HCC that are most likely to respond to these therapies. $^{3}$

HCC usually emerges in the setting of liver cirrhosis of any cause. In one analysis, hepatitis $B$ virus (HBV) or hepatitis $\mathrm{C}$ virus (HCV) is responsible for approximately $76 \%$ of the global burden of HCC, whereas approximately $24 \%$ of HCC worldwide is not virus-associated. ${ }^{4} \mathrm{HBV}$-associated $\mathrm{HCC}$ is more common in much of the developing world where there is a higher prevalence of hepatitis $B$ virus carriers. In the USA, HCC is more commonly attributed to HCV infection, alcohol use, and non-alcoholic fatty liver disease. ${ }^{5}$ The precise mechanisms of carcinogenesis in HBV, HCV, and non-viral HCC are incompletely understood. HCV-associated HCC almost invariably occurs in the setting of advanced cirrhosis and most likely arises as a result of chronic inflammation, liver regeneration, and dysplasia. ${ }^{6}$ By contrast, HBV infection can sometimes result in HCC in the absence of cirrhosis. ${ }^{8}$

We hypothesized that the different etiological HCC subsets may have a unique immune microenvironment, related to differences in disease pathogenesis and viral antigen 
expression. The immune system recognizes and can eliminate cancer primarily through the recognition of neoantigens, which are abnormal proteins not expressed on normal host cells. ${ }^{9}$ In virus-associated cancers, viral antigens expressed by tumor cells may serve as potent antigens, increasing the number of antigen-specific $\mathrm{T}$ cells and enhancing responses to immune checkpoint inhibitors. ${ }^{10}$ For example, the presence of Merkel cell polyomavirus in Merkel cell carcinoma (MCC) is associated with a robust immune infiltrate and increased tumor cell PD-L1 expression compared with virally-unassociated MCC. ${ }^{11}$ Likewise, HPV-positive head and neck squamous cell carcinoma (HNSCC) has a more extensive lymphocyte infiltrate than HPV-negative HNSCC, ${ }^{12}$ and EpsteinBarr virus (EBV)-associated gastric cancer has a more extensive lymphoid infiltrate and higher response rate to anti-PD1 immunotherapy than EBV-negative gastric cancer. ${ }^{13-18}$ Conversely, cancers resulting from oncogenic viruses may have lower mutational burdens than cancers that result from carcinogens, resulting in a lower number of mutation-associated neoantigens. To our knowledge, a comprehensive analysis of the tumor mutational burden and immune microenvironment for HCV, HBV, and uninfected HCC has not been reported previously.

Identifying differences in the immune microenvironment between virally-infected and uninfected HCC may support the development of rational immunotherapy combinations targeting specific immune modulatory signals in the various subsets of HCC, and identify patients most likely to benefit from ICI therapy. Here we perform a meta-analysis of published immunotherapy clinical trials to determine if there is a relationship between viral status and response rates to ICIs. We also compare tumor immune microenvironment features across the three cohorts using RNA expression data from The Cancer Genome Atlas (TCGA), and describe qualities of peripheral blood lymphocytes in virally associated and uninfected HCC treated with PD-1 therapy using an independent patient cohort. Our findings provide new insights into immune responses in HCC.

\section{METHODS}

\section{Systematic review and meta-analysis strategy}

We conducted an electronic searches of MEDLINE (from January 1, 2013, to January 1, 2020), as well as abstracts presented at ASCO, ESMO, AACR Annual Meetings 20122019 to identify clinical trials of anti-PD1 or anti-PD-L1 studies in HCC. We searched for clinical trials using the specific search terms HCC, plus: nivolumab, pembrolizumab, atezolizumab, durvalumab, avelumab, and cemiplimab. Only English publications were considered. We excluded trials with a total sample size or a sample size in the subgroup of interest less than 10 patients. We also excluded studies that investigated immunotherapy in combination with other classes of agents, such as combinations of ICIs with vascular endothelial growth factor (VEGF) targeted agents, because of the possibility that responses could be driven by the targeted agents rather than the ICI. However we did not exclude studies of PD-1 or PD-L1 inhibitors in combination with other systemic immunotherapies (for example, combinations of PD-1 inhibitors with CTLA-4 inhibitors). Among the studies that met our search criteria, we extracted information about objective response rates (ORR) for virally infected and uninfected patients. Among the nine studies that met our initial inclusion criteria, six of these studies provided information about response status stratified by etiology. ORR was defined as the number of responders by Response Evaluation Criteria in Solid Tumors (RECIST) V.1.1, divided by the total number of patients treated.

Meta-analysis using DerSimonian-Laird method based on a random-effect model was conducted to estimate objective response rates for trials of virally infected and uninfected patients. To estimate the difference of response rate between virally infected and uninfected patients with all trials, meta-analysis using DerSimonianLaird method via a random-effect model was utilized to compute overall difference, assuming that the set of differences is normally distributed.

\section{TCGA analysis}

Gene-level RNA sequencing (RNAseq) data was downloaded from Genomic Data Commons harmonized database for 193 HCC patients from TCGA program using the TCGAbiolinks package. ${ }^{19}$ As gene expression, we used Fragments Per Kilobase of transcript per Million mapped reads upper quartile (FPKM-UQ) that were log2tranformed for the further analysis. Mutational data for 176 HCC patients were obtained from combined public mutation annotation file created by the TCGA MC3 project. ${ }^{20}$ As a measure of tumor mutational burden (TMB), we used the total number of non-synonymous mutations per sample that were $\log 10$-transformed for the further analysis. Patient-level HBV and HCV infection calls were extracted from the supplemental table from the TCGA HCC marker paper. ${ }^{21} \mathrm{~T}$ cell receptor (TCR) and $\mathrm{B}$ cell receptor (BCR) counts were extracted from RNAseq data from TCGA with TRUST and clonotype diversity was represented as number of unique CDR3 calls in each sample per thousand reads in the TCR regions provided by Dr X Shirley Liu and Dr Bo Li. ${ }^{22}{ }^{23}$ In our analysis we used only patients that have both RNAseq and infection data, and we excluded four patients with both HBV and HCV infection $(n=189)$. To test if gene expression is associated with viral status, we applied empirical Bayes modified analysis of variance as implemented in the limma package ${ }^{24}$ in $\mathrm{R}$. The statistics from this test were used to generate a barcode plot for Th1/IFNG gene signature. A gene was considered significantly associated with viral status if adjusted $p$ value was less than 0.05 . To test if TMB, TCR and BCR counts were associated with viral status, we used an analysis of variance model as it implemented in build-in $\mathrm{R}$ aov function. In this case, the association was considered significant if $\mathrm{p}$ value was less than 0.05 . Data analysis and visualization were performed 
using R/Bioconductor software (V.3.5.0) with build-in packages and custom routines.

\section{PBMC collection, storage, and preparation for CyTOF}

A total of 21 patients with HCC treated at the Sidney Kimmel Comprehensive Cancer Center at Johns Hopkins were retrospectively identified from the Johns Hopkins Liver Cancer biobank. All of the patients received prior treatment with anti-VEGF targeted therapy, and received nivolumab or pembrolizumab (anti-PD1) therapy. To elucidate the immunologic changes that occur in peripheral blood mononuclear cells (PBMCs) with therapy in these patients, blood samples from two time points were analyzed: baseline and approximately 8 weeks after antiPD1 therapy was initiated.

Blood was collected in two BD Vacutainer CPT - Cell Preparation Tube with Sodium Heparin and processed within 2 hours of collection. Tubes were centrifuged at room temperature for approximately $30 \mathrm{~min}$ at 1800 relative centrifugal force (RCF). PBMCs were aspirated and pooled into a separate $50 \mathrm{~mL}$ conical and washed with RPMI medium. PBMCs were then counted and resuspended in AIM V and 10\% dimethylsulfoxide (DMSO) at a concentration of 5 million cells per vial. Cryovials were initially stored at $-80^{\circ} \mathrm{C}$ and transferred to liquid nitrogen for long-term storage prior to staining.

On the day of staining, peripheral blood samples were thawed rapidly in warm water bath and gently rinsed twice in RPMI media with $10 \%$ fetal bovine serum (FBS) and $1 \%$ penicillin-streptomycin according to standard protocols. Cells were rested at $37^{\circ} \mathrm{C}$ in $5 \%$ carbon dioxide for at least $30 \mathrm{~min}$ prior to further manipulation. To permit simultaneous analysis of cytokine production, all samples were stimulated in $1 \mathrm{X}$ phorbol-12-myristate 13-acetate (PMA)/ionomycin/brefeldin A cocktail (Biolegend) for 2.5 hours in complete media. During the final $15 \mathrm{~min}$ of stimulation, ethylenediaminetetraacetic acid (EDTA) was added to a final concentration of $4 \mathrm{mM}$. Cells were then rinsed again with serum-free media containing EDTA prior to staining procedures.

\section{Antibodies}

A list of cytometry by time-of-flight (CyTOF) antibodies, isotopes, and concentrations used for immune cell subtyping is listed in online supplementary table 1 . Conjugation of primary antibodies was performed using Maxpar conjugation kits according to the manufacturer's instructions. Briefly, purified CD45 antibodies were run through a buffer exchange protocol using $50 \mathrm{kDa}$ ultra filtration columns (Amicon) and then partially reduced with $4 \mathrm{mM}$ TCEP (Thermo Scientific). Polymers were loaded individually with isotopically enriched metals, 113In (Trace Sciences) and 115In (Sigma). Isotopically enriched cisplatinum $(194,198)$ were directly conjugated onto reduced CD45 antibodies. ${ }^{25}$ Antibody concentrations in the wash buffer were quantified using Nanodrop. The final antibody concentrates were then diluted in a stabilization buffer (Candor) containing 0.3\% sodium azide. Each antibody was titrated by testing a range of three to four serial dilutions utilizing positive controls (eg, stimulated PBMCs from healthy volunteers) for cytokine staining, and identifying the concentration that permits discrimination while minimizing spillover signals (maximum signal/noise ratio).

\section{CyTOF staining and data acquisition}

Approximately 1.5 million cells per sample were plated onto a 96-well plate and all samples were rinsed with phosphate-buffered saline (PBS) with $2 \mathrm{mM}$ EDTA after plating. Live/dead staining was performed with 5 min incubation in $500 \mathrm{nM}$ palladium chloride (Sigma) dissolved in DMSO and diluted in PBS, subsequently quenched with complete media. For multiplexing samples, a live cell barcoding strategy was used ${ }^{26}$ which involves CD45 monoclonal antibodies (mAbs) labeled with five different metals (89Y, 113In, $115 \mathrm{In}, 194 \mathrm{Pt}, 198 \mathrm{Pt}$ ) conjugated to CD45 antibodies for a ' 5 -choose-3' scheme for a total of 10 possible unique barcodes. Each sample was then stained with a unique barcode for $25 \mathrm{~min}$ at room temperature. Up to 10 samples were then combined into a single tube and washed with cell staining buffer (Fluidigm). Multiplexed samples were then incubated in Fc block (Invitrogen) for $10 \mathrm{~min}$ at room temperature. Surface marker staining was first done by incubating the tube in a cocktail of chemokine receptor antibodies (CCR5, CCR6, CCR7, CXCR3) for $10 \mathrm{~min}$ at $37^{\circ} \mathrm{C}$, followed by the rest of surface markers for $30 \mathrm{~min}$. After two washes, intracellular staining was performed using Cytofix/Cytoperm kit (BD Biosciences) per manufacturer's protocol. On completion of staining, cells were stored in fresh $1 \%$ methanolfree formaldehyde in PBS (Thermo Scientific) until the day of data collection. Just before data collection, all cells were labeled with rhodium (Fluidigm) at 1:1000 for $45 \mathrm{~min}$ at room temperature. All events were acquired on a Helios mass cytometer (Fluidigm). Mass cytometry data were acquired at the University of Maryland School of Medicine Center for Innovative Biomedical Resources Flow Cytometry and Mass Cytometry Core Facility, Baltimore, Maryland.

\section{CyTOF data analysis}

Randomization, bead normalization, and bead removal of data collected were performed on CyTOF software (Fluidigm) V.6.7. Using FlowJo (BD) V.10.5, single cell events were identified by gating a tight population based on cell length and rhodium signal. Dead cells were then eliminated by manually gating out cells positive for 106Pd and 108Pd on a biaxial plot. Debar coding was carried out by manual gating to select for events that are positive for the three metal-labeled CD45 mAbs assigned to the sample and negative for the remaining two metal-labeled CD45 mAbs. For all CyTOF analyzes, a computational pipeline based on diffcyt ${ }^{27}$ was employed using R V.3.5. Briefly, for unsupervised clustering, the FlowSOM algorithm ${ }^{28}$ was used to identify 30 meta-clusters that were then annotated into specific immune cell subtypes. Clustering was 
visualized using a two-dimensional uniform manifold approximation and projection (UMAP) dimensionality reduction algorithm. ${ }^{29}$ Two thousand cells per sample were used for visualization. For differential analyzes, negative binomial methodology was used for cell type abundance comparisons (edgeR) and linear mixed modeling was used for mean marker intensity comparisons (limma).$^{30}{ }^{31}$ False discovery rate of $10 \%$ (adjusted $\mathrm{p}$ value $<0.1)$ were denoted as statistically significant in the figures. The final model for differential analyzes incorporated random batch effects. Both standard per-cell basis UMAP and schex-computed hexbins (https://github. com/SaskiaFreytag/schex) within the UMAP were used to reveal any overt batch-specific biases (online supplementary figure 1 ).

\section{RESULTS}

\section{Meta-analysis of response rate by viral status}

We first investigated whether there was a difference in clinical response rate for immune checkpoint therapy by viral status in HCC. A total of nine studies met our initial criteria for immune checkpoint therapy in HCC, of which six studies had stratified response information by viral status (uninfected, or virally associated (HBV, HCV, or coinfection)). These included studies of anti-PD1 therapy (nivolumab and pembrolizumab), anti-PD-L1 therapy (durvalumab), and anti-PD-(L) 1 in combination with anti-CTLA-4 (tremelimumab, ipilimumab). Collectively, 567 patients were included on these studies across multiple different geographical areas across the world. Across the studies, clinical activity was observed across both virally infected and uninfected patients (table 1).

Random-effects meta-analysis was performed to obtain the response rate from trials of viral positive and negative patients. The response rate obtained by meta-analysis was $19.2 \%$ (95\% CI: $14.5 \%$ to $23.9 \%$ ) and $17.7 \%(95 \%$ CI: $6.8 \%$ to $28.6 \%$ ) among uninfected and infected patients, respectively (online supplementary figure 2). Subsequently, random-effects meta-analysis was utilized to estimate the difference of response rate between the virally infected and uninfected HCC patients. The absolute difference of response rate is $-1.4 \%$ (95\% CI: $-13.5 \%$ to $10.6 \%$ ), which is not clinically significant.

Multiple studies included in our meta-analysis enrolled multiple patients with $\mathrm{HCV} / \mathrm{HBV}$ co-infection, and therefore our ability to distil responses into viral subtype (HBV vs HCV) was limited. However response rates were similar in HBV and HCV infected patients. In the CheckMate 040 phase 2 study of nivolumab monotherapy and nivolumab plus ipilimumab, HCV patients responded at a numerically higher rate than HBV patients, whereas in the Keynote 224 phase 2 study HBV patients responded at a numerically higher rate than HCV patients.

\section{Tumor immune microenvironment in HCV, HBV, and uninfected HCC}

We subsequently utilized gene-level RNAseq data from TCGA to investigate potential differences in the tumor immune microenvironment in virally infected and uninfected patients. Even though virally infected and uninfected patients responded similarly to anti-PD-1/PD-L1 therapy, we hypothesized that the mechanism of response could be different across the major HCC etiologic groups, reflecting differences in the tumor immune microenvironment. We first looked at the effect of etiology (HBV, HCV, and uninfected HCC) on tumor infiltrating lymphocytes. A high baseline intratumoral immune cell infiltration may improve response likelihood to anti-PD-1 therapies, and cytotoxic CD8 T cells density within the tumor microenvironment in particular is thought to be an important pan-tumor determinant of ICI therapy response. ${ }^{29} \mathrm{We}$ found no association between etiology and expression of key markers of $\mathrm{CD} 8+\mathrm{T}$ cells $(C d 8 a), \mathrm{CD} 4+\mathrm{T}$ cells $(C d 4)$, CD20 + B cells (Ms4a1), or CD68 + macrophages (Cd68) (all $\mathrm{p}>0.05$; figure 1 ).

Expression of PD-L1 on tumor or immune cells within the tumor microenvironment (TME) have been associated with response to ICI therapy in multiple tumor types including HCC. Therefore we examined the relationship

Table 1 A total of six studies, collectively reporting on the results of 567 patients, were used for meta-analysis. these included studies of anti-PD1 therapy (nivolumab and pembrolizumab), anti-PD-L1 therapy (duralumab), and anti-CTLA-4 therapy (ipilimumab and tremelimumab). Across the studies, clinical activity was observed across both virally infected and uninfected patients

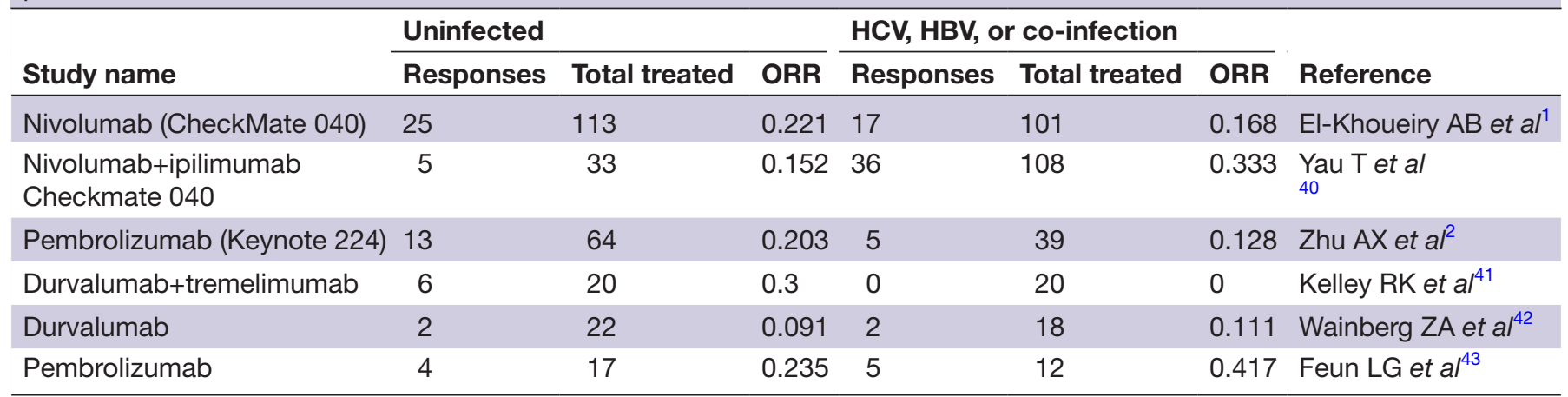

$\mathrm{HBV}$, hepatitis B virus; HCV, hepatitis C virus; ORR, objective response rate. 

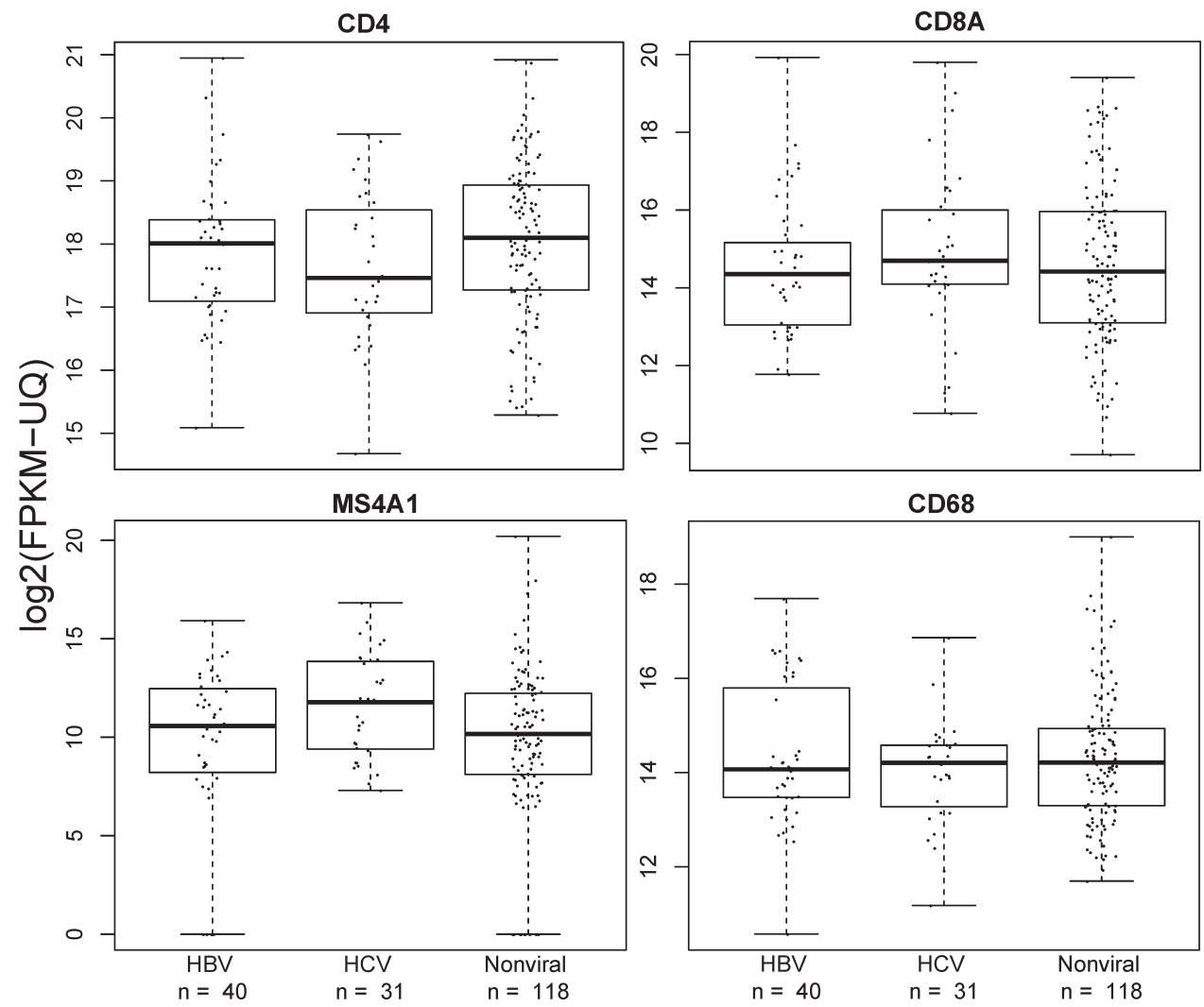

Figure 1 There was no significant association between hepatocellularcarcinoma etiology and immune infiltration using The Cancer Genome Atlas RNA expression data. HBV, hepatitis B virus; HCV, hepatitis C virus.

between HCC etiology (HBV, HCV, and uninfected HCC) and expression of PD-L1 and other immune checkpoint molecules such as PD-1, LAG3 and CTLA4, within the TME. The expression of immune checkpoint molecules were similar across the three major etiologic subtypes, again showing no significant effect of etiology on immune-related biomakers (figure 2, and online supplementary figure 3). We next looked at whether HCC etiology was associated with genes associated with a Th1/IFN- $\gamma$-related immune signature. The presence of a Th1/IFN- $\gamma$ gene signature at baseline has previously been associated with improved response to ICI therapy. ${ }^{21}$ For this analysis we combined genes from the published Th1 signature from Gentleman and colleagues, ${ }^{32}$ and genes
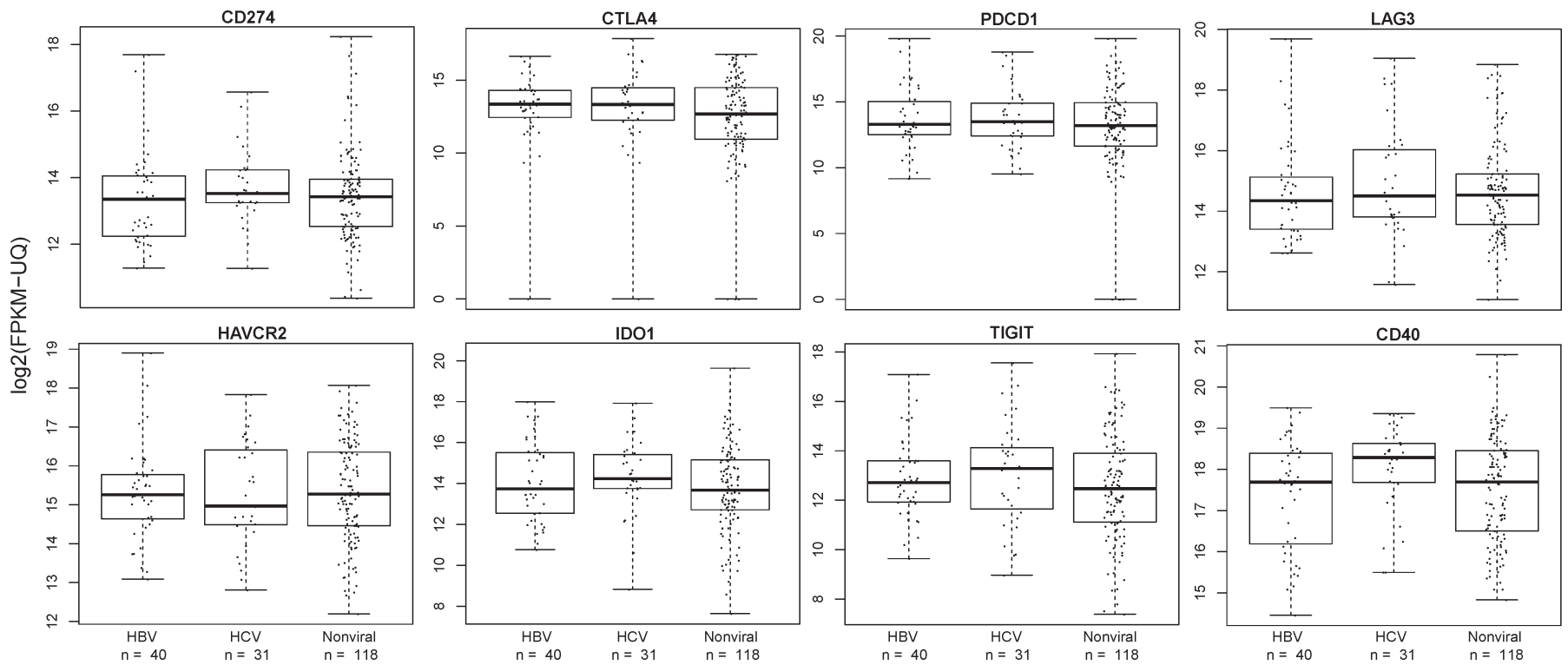

Figure 2 There was no significant association between hepatocellularcarcinoma etiology and expression of immune markers within the tumor microenvironment using The Cancer Genome Atlas RNA expression data, including expression of PD-L1 (CD274), PD-1 (PDCD1), CTLA4, and LAG3. 


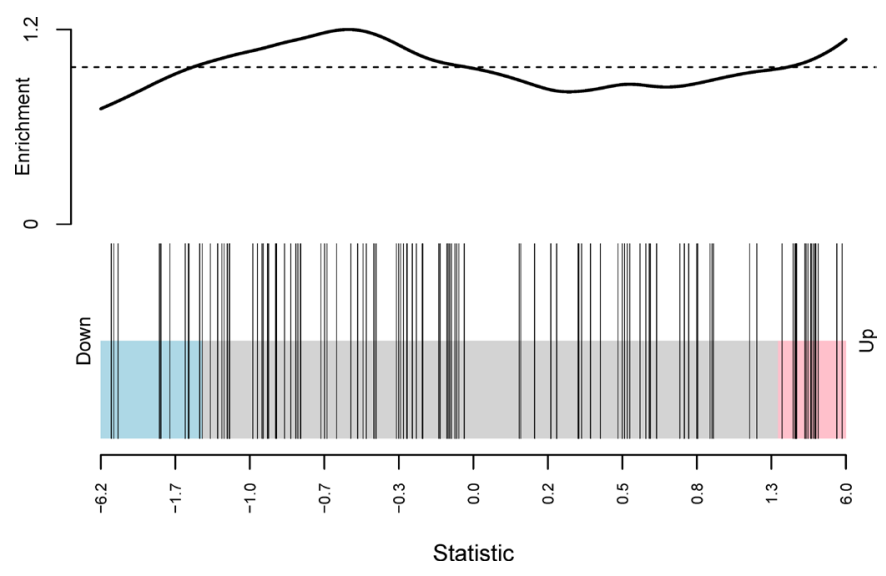

Figure 3 There was no significant enrichment in Th1/IFN- $\gamma$-related immune signature genes that are differentially expressed between the viral and non-viral hepatocellularcarcinoma etiologies., using The Cancer Genome Atlas RNA expression data. The vertical bars represent statistics of association of gene expression with viral status. The statistics is from the empirical Bayes modified analysis of variance as implemented in the limma package. ${ }^{24}$ On the $y$-axis, there is the relative enrichment of the vertical bars.

from IFN $\gamma$ signaling pathway from the Reactome database (http:/ / www.reactome.org) ${ }^{33}$ Again, we found that HCC etiology had no significant effect on the expression of genes within this signature that may be predictive of PD-1 response (figure 3 and online supplementary figure 4).

We additionally looked beyond immune-related molecules to see if there were differences in the TMB between virally associated and unassociated HCC. We and others have previously shown that the TMB, a surrogate for the number of mutation-associated cancer neoantigens, are associated with a higher response to ICI ${ }^{10}$ and that the TMB provides independent information from PD-L1

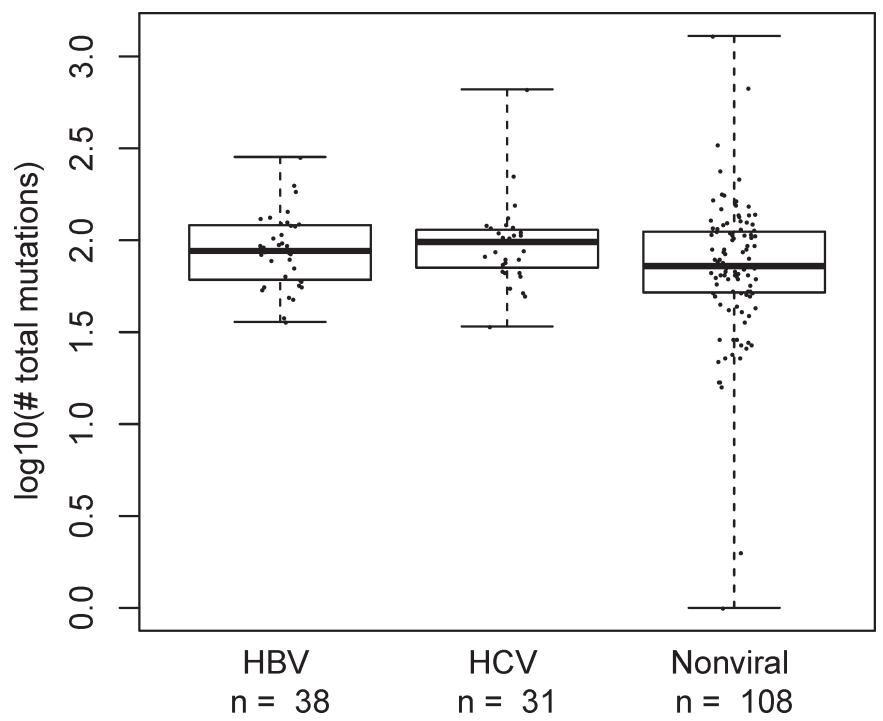

Figure 4 The tumor mutational burden was similar in virallyinfected and uninfected hepatocellularcarcinoma, using DNA mutation data from the The Cancer Genome Atlas. HBV, hepatitis $B$ virus; $\mathrm{HCV}$, hepatitis $\mathrm{C}$ virus. expression in multiple tumor types. ${ }^{34}$ We found no significant effect of HCC etiology on the TMB ( $p>0.05$; figure 4). Finally, we analyzed tissue-resident TCRs and BCRs estimated from bulk RNAseq data in TCGA to see if there was an association between HCC etiology and the diversity of TCRs and BCRs within the tumor microenvironment. ${ }^{22}{ }^{23}$ We hypothesized that the presence of chronic viral antigens within the tumor microenvironment would lead to decreased TCR and BCR diversity, which account for any observed differences in anti-PD1 response rates. However, we found no significant effect ( $p>0.05)$ of HCC etiology on TCR or BCR diversity within tumor immune lymphocyte populations (figure 5).

\section{Comparing immunologic responses in virus and non-Virus associated HCC}

To identify any potential differences in the immunologic response to PD1 pathway targeting between virus and non-virus associated HCC, we also compared the differences between baseline and 8-week post-treatment lymphoid immune profiles in the peripheral blood by mass cytometry (CyTOF, that is, cytometry by time-offlight). We retrospectively identified 21 patients with available peripheral blood samples, 10 with non-viral and 11 with viral etiologies of HCC, respectively, at Sidney Comprehensive Cancer Center at Johns Hopkins. All patients were treated with pembrolizumab or nivolumab at standard approved dosages, and had received at least one VEGF-targeted therapy prior to anti-PD1 treatment. Six of the 21 patients demonstrated clinical responses, three of which had virus associated HCC. Given the limited number of samples, analysis was focused on interrogating the immunologic responses to treatment without clinical correlations. A total of 42 samples were stained in a multiplexed fashion using a $\mathrm{T}$ cell-oriented CyTOF panel of 27 markers (online supplementary table 1). To determine subtypes, 17 markers were used to perform unsupervised clustering analysis via FlowSOM algorithm. Clustering analysis identified 30 meta-clusters, which were then annotated into 19 subtypes of T cells, 2 subtypes of B cells, 4 subtypes of NK cells, and 4 indeterminate immune cell subtypes (online supplementary figure 5 , table 2 ). In our HCC cohort, among all the immune cell clusters, anti-PD1 pathway treatment significantly impacted the proportions of only two clusters in the peripheral blood: a cluster of cytotoxic $\mathrm{T}$ cells with effector memory phenotype ('Tc EM 2') increased and a cluster of B cells ('B cells 2') decreased (figure 6, online supplementary figure 6). Overall trend in the $\mathrm{T}$ cell profiles favored a shift toward memory phenotypes. On stratification by viral status, no apparent differences were observable (figure 6).

Functional profiles within the defined immune cell clusters was assessed by analyzing the following types of markers: proliferation state (Ki67), cytolytic function (Granzyme B), cytokine production (IL2, TNF $\alpha$, IFN $\gamma$ ), checkpoint/co-inhibitory markers (PD1, CTLA4, TIM3, Lag3), co-stimulatory markers (41BB, OX40). The mean metal intensities for each of the markers within each 

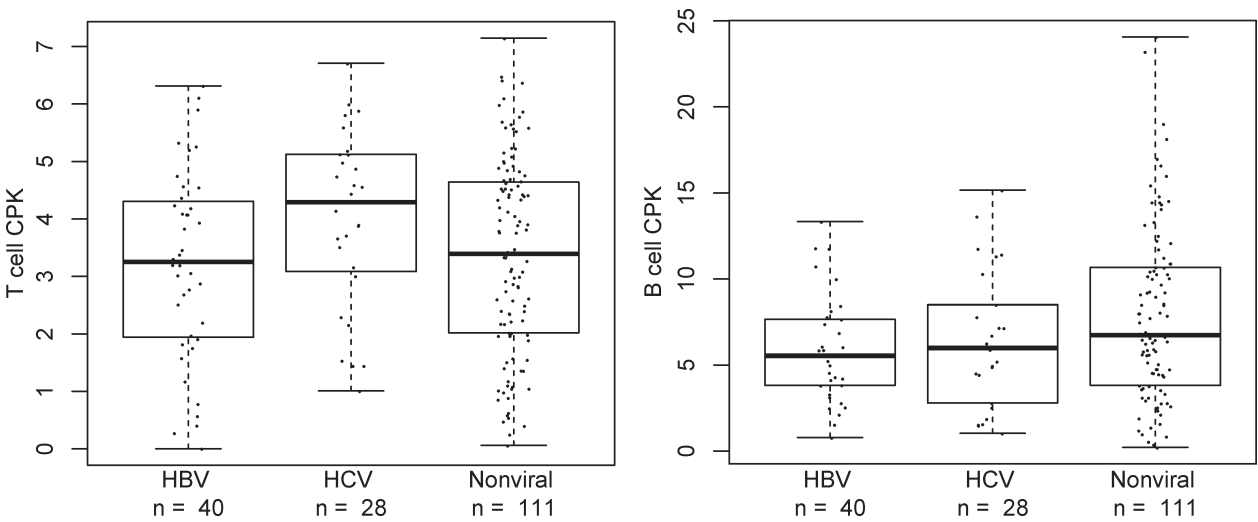

Figure 5 Counts of unique CDR3 per thousand of tissue-resident (A) T cell repertoires (TCRs) and (B) B cell repertoires (BCRs) derived from The Cancer Genome Atlas RNA expression data showed no significant association between hepatocellularcarcinoma etiology and TCR and BCR diversity within the tumor microenvironment. HBV, hepatitis B virus; HCV, hepatitis $\mathrm{C}$ virus.
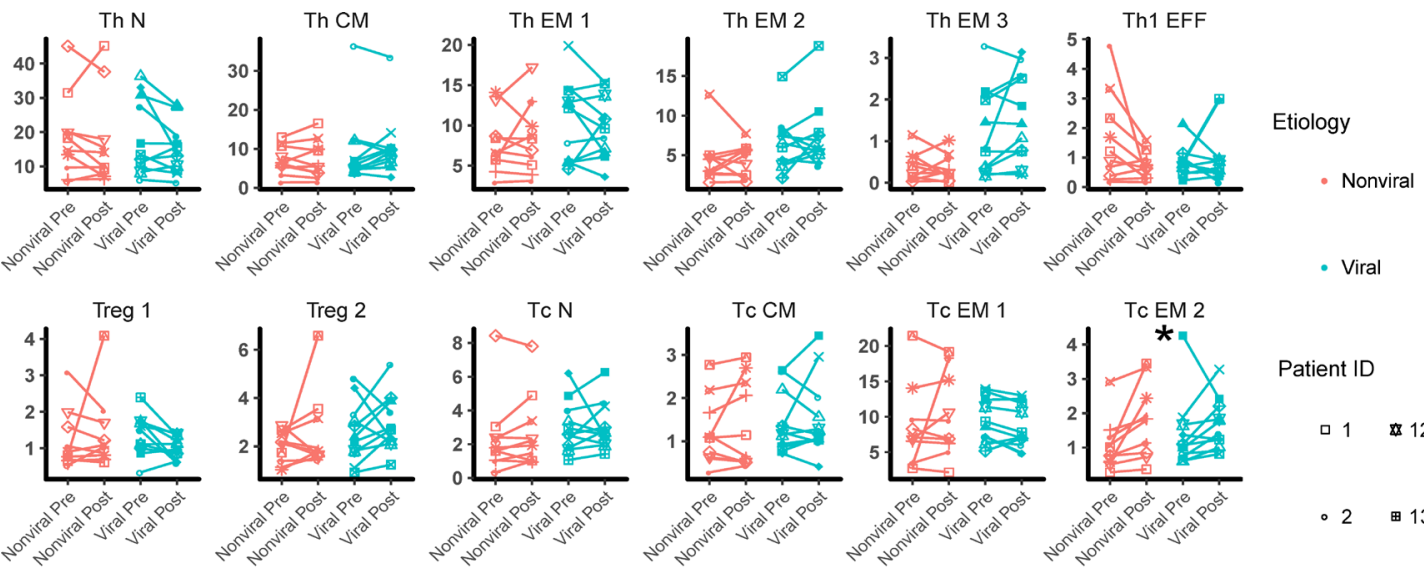

Patient ID
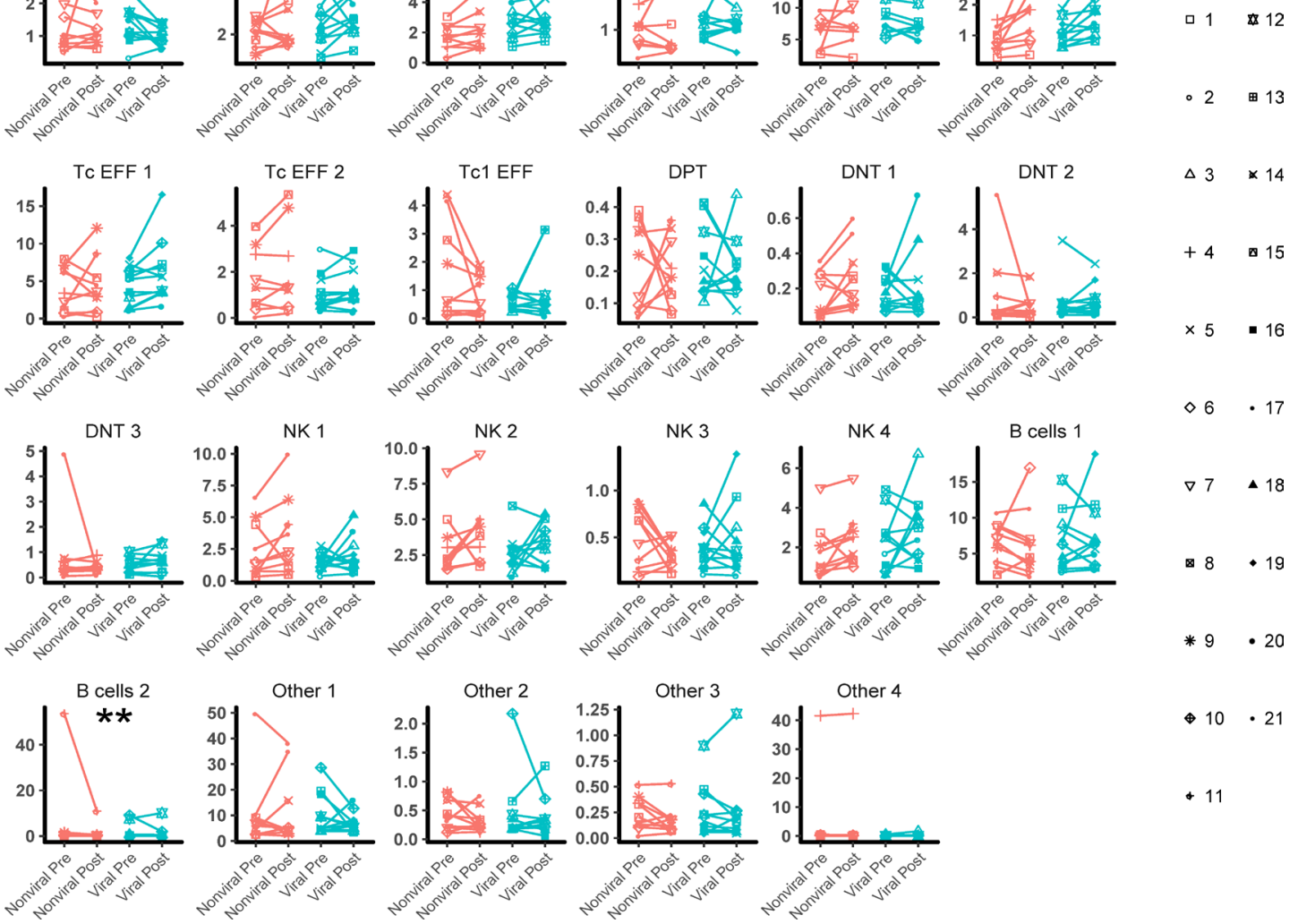

Figure 6 Changes in the immune cell subtypes with PD1 therapy in HCC patients. Alteration in the proportion (\% of CD45) of immune cell subtypes in the peripheral blood with anti-PD1 pathway treatment are shown in line plots connecting baseline ('pre') and after treatment ('post') conditions. Individual-specific changes for each of the 21 patients is denoted by a unique marker shape. Patients are also stratified by non-virus (red) and virus (blue) etiologies of HCC. FDR-adjusted ${ }^{\star} \mathrm{p}<0.05$ and ${ }^{* *} \mathrm{p}<0.01$ when comparing pre-treatment and post-treatment conditions are shown. HCC, hepatocellular carcinoma. 


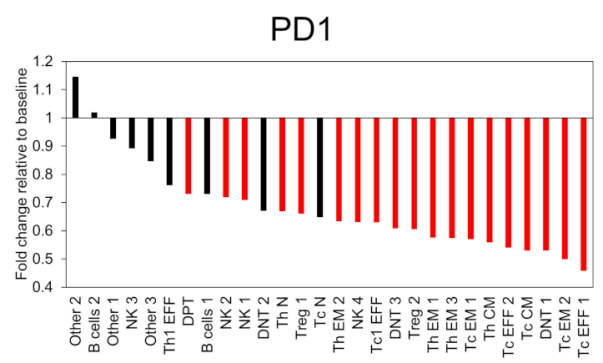

TIM3

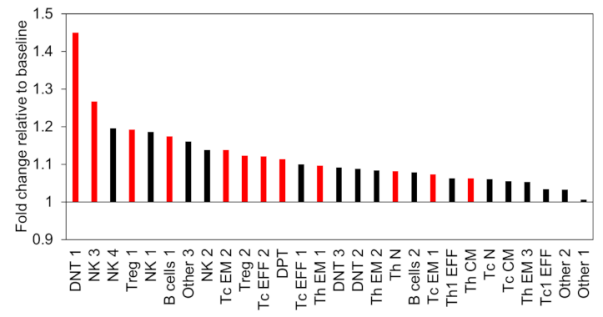

OX40

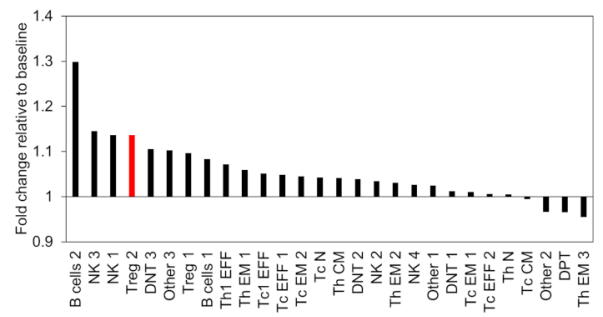

TNFa

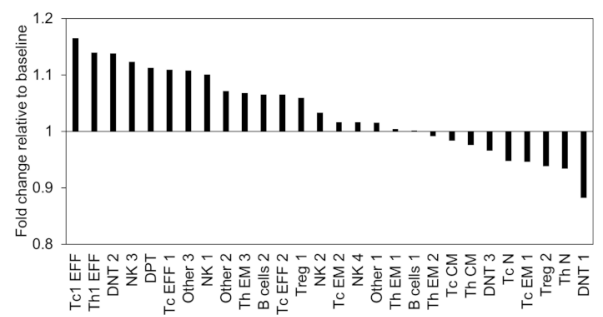

CTLA4

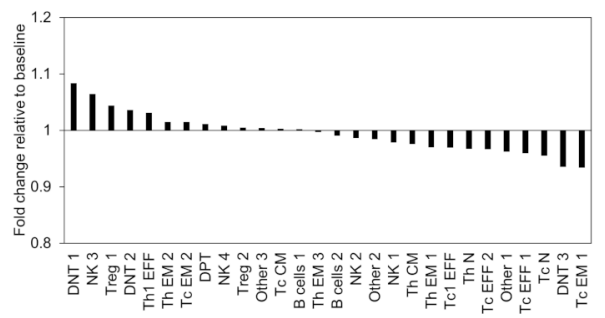

Ki67

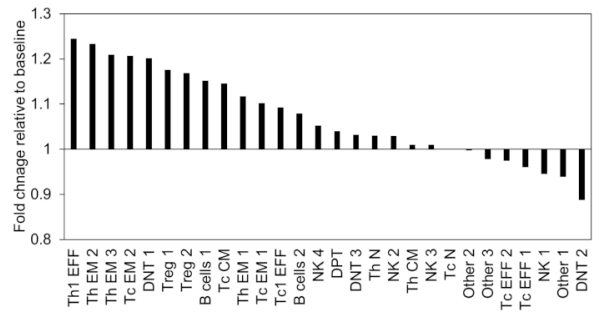

41BB

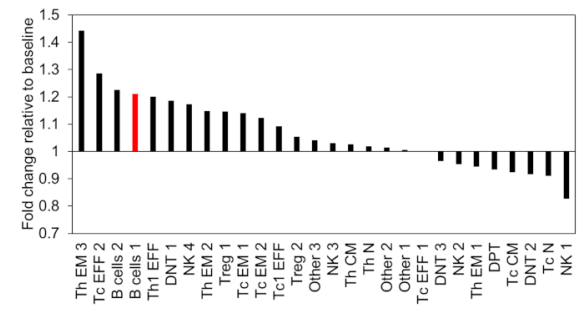

IFNg

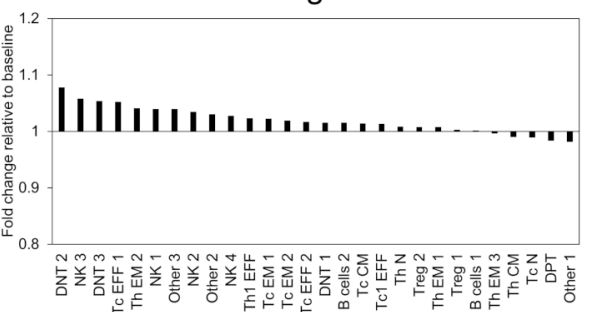

Lag3

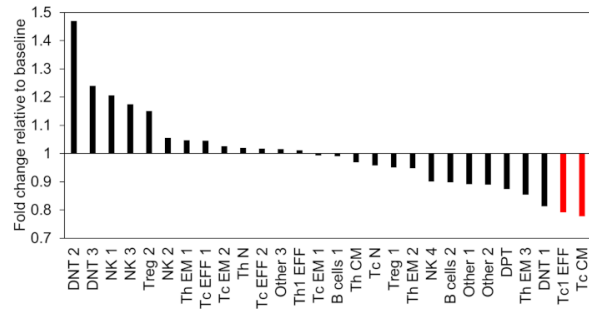

Granzyme B

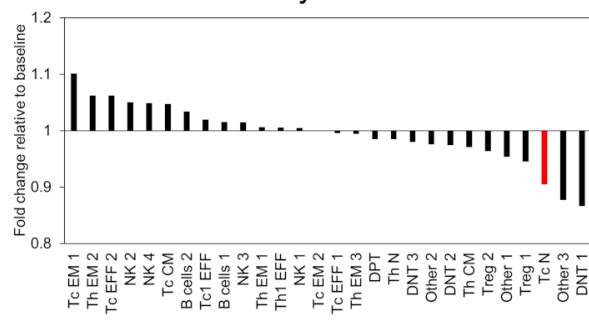

IL2

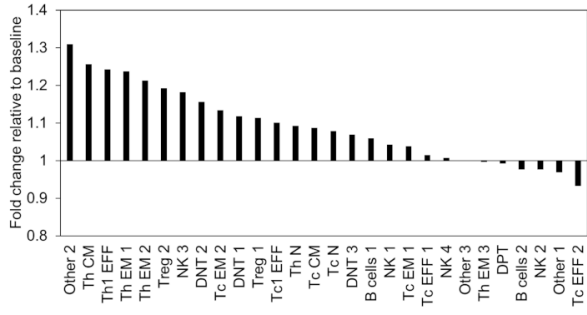

$P_{\text {adj }}<0.1$

n.s.

Figure 7 Changes in the functional markers with PD1 therapy in hepatocellularcarcinoma patients. Average fold change with PD1 therapy relative to baseline in each of the functional markers within each of the immune cell type are shown. Cell types are sorted across the horizontal axis based on the highest to lowest fold change. Fold changes with FDR-adjusted $p$ values $<0.1$ are marked in red.

cluster before and after anti-PD1 pathway therapy were compared, and the impact of viral status on the effect of treatment was also assessed. As expected, consistently lower PD1 expression was demonstrated in post-treatment samples. This provided a technical validation for patients who received anti-PD1 antibodies, demonstrating a PD1specific occupancy effect. Notably, treatment with antiPD1 pathway significantly increased TIM3 expression in many subtypes and led to trends toward increased Ki67, IL2, OX40, and 41BB (figure 7, online supplementary figure 7). Virus association did not have significant (FDR adjusted $\mathrm{p}<0.05$ ) effects on any of these immunological changes (online supplementary table 3 ). Comparing only the pretreatment profiles, however, patients with positive viral status exhibited higher abundance of memory phenotypes (online supplementary figure 8), higher expression levels of PD1, TIM3, TNF $\alpha$, and IFN $\gamma$, and lower expression levels of $41 \mathrm{BB}$ and $\mathrm{Ki}-67$ within some of the $\mathrm{T}$ cell clusters, especially helper $\mathrm{T}$ cells (online supplementary figure 9). Taken together, the results verify positive immunological changes to PD1 therapy consistent with enhanced antitumor effects in HCC patients and their occurrence irrespective of the patients' virally associated pretreatment immunological states.

\section{DISCUSSION}

In summary, we find that both virally infected and uninfected HCC respond similarly to inhibitors of the PD-1/ PD-L1 axis. Multiple completed studies of PD-1/PD-L1 therapy alone or in combination with other agents will likely be reported in the next few years, ${ }^{3}$ which will further clarify the relationship between HCC etiology and clinical response to these agents. However, our results suggest that viral status should not be used clinically to identify patients for treatment with PD-1/PD-L1 inhibitor therapy. 
We were unable to include objective response information from two large phase 3 studies (Keynote-240 and Checkmate-459) in our meta-analysis, because the published reports of these studies did not break down objective response rates by etiology. However in both of these phase 3 studies, clinical benefit was consistent across hepatitis infectious status, ${ }^{35}{ }^{36}$ further supporting the overall conclusion of our meta-analysis. The results of our clinical trial meta-analysis are supported by TCGA data and peripheral blood samples from an independent cohort of patients at Johns Hopkins Hospital that collectively show no significant association between HCC etiology and widely utilized immune-related biomarkers such as expression of CD8, PD-L1, a Th1/IFN- $\gamma$-related immune signature, or mutational burden. Such results are also in agreement with a prior analysis of the HCC tumor microenvironment by our group using immunohistochemistry that similarly showed no significant difference in immune infiltrate by etiologic subtype ${ }^{37}$ In the present study we did not look specifically at minor etiological subtypes, such as HCC induced by dietary aflatoxin intake, which has previously shown a strong relationship with specific genomic alterations. ${ }^{38}$ For this reason, we cannot exclude the possibility that some smaller and distinct subtypes of HCC may be associated with particular immunologic features or responses to inhibition of the PD-1/PD-L1 axis. However our results broadly support the conclusion that HCC viral etiology has little or no effect on common immune-related biomarkers within the TME.

It is unclear why, in contrast to multiple other tumor types that have viral and non-viral etiologic subsets, virallyinfected HCC does not differ significantly with regard to the tumor immune microenvironment or responses to PD-1/PD-L1 inhibitor therapy. It is well demonstrated that ICI therapy can promote antiviral immunity leading to control of viral hepatitis, ${ }^{39}$ and therefore it is rational to hypothesize that viral antigens within the tumor microenvironment could modulate the anti-tumor immune response in the context of ICI therapy. However, in contrast to some tumor types such as MCC in which viral antigens are largely restricted to tumor cells, HBV and HCV can chronically integrate into both tumor cells and normal hepatocytes in the background liver. Therefore, virus-specific $\mathrm{T}$ cells may be unable to discriminate between virally-infected hepatocytes and virally-infected tumor cells, and may therefore fail to augment anti-tumor immunity in HCC. In other words, carcinogenesis of virally associated hepatocellular carcinoma may be driven by the underlying cirrhosis rather than the preceding virus-specific immune responses. Our cytometric analyzes suggested that while hepatitis virus infections were associated positive immune responses detectable in the peripheral blood, that is, increased memory $\mathrm{T}$ cell populations at baseline, they are ultimately not HCC-specific. This interpretation is consistent with our finding that anti-PD1 therapy did not leverage the differences in the baseline immunological states into significantly enhanced clinical antitumor responses.
A major limitation of our clinical meta-analysis is that we use aggregated clinical trial data and are therefore unable to adjust for potential covariates that may impact responses to PD1 inhibitor therapy, such as geographical region, ethnicity, age, or other factors. Additionally, the use of sample cohorts from the USA may not fully reflect the HCC biology globally. Strengths of our investigation include the use of both tumor data and peripheral blood samples to evaluate the relationship of HCC etiology and responses to PD-1/PD-L1 inhibitor therapy. In summary, in contrast to other tumors with virally-associated and unassociated subsets, we find no major effect of HCC etiology on multiple immune-related biomarkers that are known to modulate responses to PD-1 inhibitor therapy. Viral status should not be used to identify patients with HCC for PD-1/PD-L1 therapy, and additional work is needed to identify other biomarkers that can more effectively identify patients for ICI therapy in HCC.

\section{Author affiliations}

${ }^{1}$ Sidney Kimmel Comprehensive Cancer Center, Johns Hopkins University School of Medicine, Baltimore, Maryland, USA

${ }^{2}$ Bloomberg-Kimmel Institute for Cancer Immunotherapy, Johns Hopkins University School of Medicine, Baltimore, Maryland, USA

${ }^{3}$ Division of Biostatistics and Bioinformatics, Johns Hopkins Medicine Sidney Kimmel Comprehensive Cancer Center, Baltimore, Maryland, USA

${ }^{4}$ Center for Vaccine Development, University of Maryland, Baltimore, Maryland, USA ${ }^{5}$ Molecular Microbiology and Immunology, University of Maryland School of Medicine, Baltimore, Maryland, USA

${ }^{6}$ Institute for Computational Medicine, Mathematical Institute for Data Science, Johns Hopkins University, Baltimore, MD, United States

${ }^{7}$ Pancreatic Cancer Precision Medicine Program, Skip Viragh Center for Pancreatic Cancer, Johns Hopkins University School of Medicine, Baltimore, MD, United States

Acknowledgements The authors are grateful to the patients who provided biospecimens that were used in this study. We thank Xiaole Shirley Liu, Bo Li, and Xiahao Hu for support in providing TCR and BCR data from TCGA samples.

Contributors WJH was responsible for experiment design, data acquisition, data analysis, data interpretation, and manuscript writing and review. LD was responsible for data acquisition, data analysis, data interpretation, and manuscript review and editing. SJL and HW performed data analysis, data interpretation, and manuscript review and editing. RV performed data analysis and manuscript review and editing. SX and JML were responsible for data acquisition and manuscript review and editing. MS was responsible for supervision of data acquisition and manuscript review and editing. EJF was responsible for data acquisition, supervision of data analysis, and manuscript review and editing. EMJ was responsible for supervision of data interpretation and manuscript review and editing. MY was responsible for conceptualization, supervision, funding acquisition, experiment design, data acquisition, data analysis, data interpretation, manuscript writing and review.

Funding Funding was provided by the National Cancer Institute (NCI) Multiscale Modeling Consortium grant (U01 CA212007), NIH T32CA00971-38 (to WJH), R50CA243627 (LD), the Johns Hopkins Bloomberg-Kimmel Institute for Cancer Immunotherapy, the Viragh Foundation, $\mathrm{NCl}$ Specialized Program of Research Excellence (SPORE) in Gastrointestinal Cancers (P50 CA062924), and the NIH Center Core Grant (P30 CA006973).

Competing interests No, there are no competing interests.

Patient consent for publication Not required.

Ethics approval Ethics approval and consent to participate: The evaluation of banked peripheral blood mononuclear cells was performed in accordance with the protocols approved by the Johns Hopkins Institutional Review Board. All peripheral blood specimens were obtained with written patient consent (IRB number CIR00051274).

Provenance and peer review Not commissioned; externally peer reviewed. 
Data availability statement All data relevant to the study are included in the article or uploaded as supplementary information. Data in the study does not include any patient identifiable data.

Open access This is an open access article distributed in accordance with the Creative Commons Attribution Non Commercial (CC BY-NC 4.0) license, which permits others to distribute, remix, adapt, build upon this work non-commercially, and license their derivative works on different terms, provided the original work is properly cited, appropriate credit is given, any changes made indicated, and the use is non-commercial. See http://creativecommons.org/licenses/by-nc/4.0/.

\section{ORCID iD}

Won Jin Ho http://orcid.org/0000-0003-2644-5086

\section{REFERENCES}

1 El-Khoueiry AB, Sangro B, Yau T, et al. Nivolumab in patients with advanced hepatocellular carcinoma (CheckMate 040): an open-label, non-comparative, phase $1 / 2$ dose escalation and expansion trial. Lancet 2017;389:2492-502.

2 Zhu AX, Finn RS, Edeline J, et al. Pembrolizumab in patients with advanced hepatocellular carcinoma previously treated with sorafenib (KEYNOTE-224): a non-randomised, open-label phase 2 trial. Lancet Oncol 2018;19:940-52.

3 Yarchoan M, Agarwal P, Villanueva A, et al. Recent developments and therapeutic strategies against hepatocellular carcinoma. Cancer Res 2019;79:4326-30.

4 Maucort-Boulch D, de Martel C, Franceschi S, et al. Fraction and incidence of liver cancer attributable to hepatitis $B$ and $C$ viruses worldwide. Int J Cancer 2018;142:2471-7.

5 El-Serag HB, Kanwal F. Epidemiology of hepatocellular carcinoma in the United States: where are we? where do we go? Hepatology 2014;60:1767-75.

6 Lok AS, Seeff LB, Morgan TR, et al. Incidence of hepatocellular carcinoma and associated risk factors in hepatitis C-related advanced liver disease. Gastroenterology 2009;136:138-48.

7 El-Serag HB, Rudolph KL. Hepatocellular carcinoma: epidemiology and molecular carcinogenesis. Gastroenterology 2007;132:2557-76.

8 Michielsen P, Ho E. Viral hepatitis B and hepatocellular carcinoma. Acta Gastroenterol Belg 2011;74:4-8.

9 Yarchoan M, Johnson BA, Lutz ER, et al. Targeting neoantigens to augment antitumour immunity. Nat Rev Cancer 2017;17:209-22.

10 Yarchoan M, Hopkins A, Jaffee EM. Tumor mutational burden and response rate to PD-1 inhibition. N Engl J Med 2017;377:2500-1.

11 Lipson EJ, Vincent JG, Loyo M, et al. Pd-L1 expression in the Merkel cell carcinoma microenvironment: association with inflammation, Merkel cell polyomavirus and overall survival. Cancer Immunol Res 2013;1:54-63.

12 Mandal R, Senbabaoğlu Y, Desrichard A, et al. The head and neck cancer immune landscape and its immunotherapeutic implications. JCI Insight 2016;1:e89829.

$13 \mathrm{Ma} \mathrm{J}$, Li J, Hao Y, et al. Differentiated tumor immune microenvironment of Epstein-Barr virus-associated and negative gastric cancer: implication in prognosis and immunotherapy. Oncotarget 2017;8:67094-103.

14 Chang MS, Lee HS, Kim CW, et al. Clinicopathologic characteristics of Epstein-Barr virus-incorporated gastric cancers in Korea. Pathol Res Pract 2001;197:395-400.

15 Gulley ML, Pulitzer DR, Eagan PA, et al. Epstein-Barr virus infection is an early event in gastric carcinogenesis and is independent of $\mathrm{Bcl}-$ 2 expression and p53 accumulation. Hum Pathol 1996;27:20-7.

16 Tokunaga M, Land CE, Uemura Y, et al. Epstein-Barr virus in gastric carcinoma. Am J Pathol 1993;143:1250-4.

17 van Beek J, zur Hausen A, Snel SN, et al. Morphological evidence of an activated cytotoxic T-cell infiltrate in EBV-positive gastric carcinoma preventing lymph node metastases. Am J Surg Pathol 2006;30:59-65.

18 Derks S, Liao X, Chiaravalli AM, et al. Abundant PD-L1 expression in Epstein-Barr virus-infected gastric cancers. Oncotarget 2016;7:32925-32.

19 Colaprico A, Silva TC, Olsen C, et al. TCGAbiolinks: an R/ Bioconductor package for integrative analysis of TCGA data. Nucleic Acids Res 2016;44:e71
20 Ellrott K, Bailey MH, Saksena G, et al. Scalable open science approach for mutation calling of tumor Exomes using multiple genomic pipelines. Cell Syst 2018;6:271-81.

21 Cancer Genome Atlas Research Network. Electronic address: wheeler@bcm.edu, Cancer Genome Atlas Research NetworkAlly A, Balasundaram M, Carlsen R. Comprehensive and integrative genomic characterization of hepatocellular carcinoma. Cell 2017;169:e23:1327-41.

22 Li B, Li T, Pignon J-C, et al. Landscape of tumor-infiltrating T cell repertoire of human cancers. Nat Genet 2016;48:725-32.

$23 \mathrm{Hu} \mathrm{X}$, Zhang J, Wang J, et al. Landscape of B cell immunity and related immune evasion in human cancers. Nat Genet 2019;51:560-7.

24 Smyth GK, Smyth GK. Limma: linear models for microarray data. In: Gentlem R, Carey V, Dudoit S, et al, eds. Bioinformatics and computational biology solutions using $R$ and Bioconductor. 420, 2005.

25 Mei HE, Leipold MD, Maecker HT. Platinum-conjugated antibodies for application in mass cytometry. Cytometry A 2016;89:292-300.

26 Hartmann FJ, Simonds EF, Bendall SC. A universal live cell Barcoding-Platform for multiplexed human single cell analysis. Sci Rep 2018;8:10770.

27 Weber LM, Nowicka M, Soneson C, et al. diffcyt: differential discovery in high-dimensional cytometry via high-resolution clustering. Commun Biol 2019;2:183.

28 Van Gassen S, Callebaut B, Van Helden MJ, et al. FlowSOM: using self-organizing maps for visualization and interpretation of cytometry data. Cytometry A 2015;87:636-45.

29 Becht E, McInnes L, Healy J, et al. Dimensionality reduction for visualizing single-cell data using UMAP. Nat Biotechnol 2019;37:38-44.

30 Ritchie ME, Phipson B, Wu D, et al. limma powers differential expression analyses for RNA-sequencing and microarray studies. Nucleic Acids Res 2015;43:e47

31 Robinson MD, McCarthy DJ, Smyth GK. edgeR: a Bioconductor package for differential expression analysis of digital gene expression data. Bioinformatics 2010;26:139-40.

32 Gentleman RC, Carey VJ, Bates DM, et al. Bioconductor: open software development for computational biology and bioinformatics. Genome Biol 2004:5:R80.

33 Croft D, O'Kelly G, Wu G, et al. Reactome: a database of reactions, pathways and biological processes. Nucleic Acids Res 2011;39:D691-7.

34 Yarchoan M, Albacker LA, Hopkins AC, et al. Pd-L1 expression and tumor mutational burden are independent biomarkers in most cancers. JCI Insight 2019;4. doi:10.1172/jci.insight.126908. [Epub ahead of print: 21 Mar 2019].

35 Sangro B, Park J-W, Dela Cruz CM, et al. A randomized, multicenter, phase 3 study of nivolumab vs sorafenib as first-line treatment in patients (PTS) with advanced hepatocellular carcinoma (HCC): CheckMate-459. J Clin Oncol 2016;34:TPS4147.

36 Finn RS, Ryoo B-Y, Merle P, et al. Pembrolizumab as second-line therapy in patients with advanced hepatocellular carcinoma in KEYNOTE-240: a randomized, double-blind, phase III trial. J Clin Oncol 2020;38:193-202.

37 Yarchoan M, Xing D, Luan L, et al. Characterization of the immune microenvironment in hepatocellular carcinoma. Clin Cancer Res 2017:23:7333-9.

38 Zhang W, He H, Zang M, et al. Genetic features of AflatoxinAssociated hepatocellular carcinoma. Gastroenterology 2017;153:249-62.

39 Sangro B, Gomez-Martin C, de la Mata M, et al. A clinical trial of CTLA-4 blockade with tremelimumab in patients with hepatocellular carcinoma and chronic hepatitis C. J Hepatol 2013:59:81-8.

40 Yau T, Kang Y-K, Kim T-Y, et al. Nivolumab (NIVO) + ipilimumab (IPI) combination therapy in patients (PTS) with advanced hepatocellular carcinoma (aHCC): results from CheckMate 040. JCO 2019;37:4012

41 Kelley RK, Abou-Alfa GK, Bendell JC, et al. Phase I/II study of durvalumab and tremelimumab in patients with unresectable hepatocellular carcinoma (HCC): phase I safety and efficacy analyses. JCO 2017;35:4073

42 Wainberg ZA, Segal NH, Jaeger D, et al. Safety and clinical activity of durvalumab monotherapy in patients with hepatocellular carcinoma (HCC). JCO 2017;35:4071

43 Feun LG, Li Y-Y, Wu C, et al. Phase 2 study of pembrolizumab and circulating biomarkers to predict anticancer response in advanced, unresectable hepatocellular carcinoma. Cancer 2019:125:3603-14. 